\title{
Metallic Concepts for Repair of Reinforced Carbon-Carbon Space Shuttle Leading Edges
}

\author{
Frank Ritzert and James Nesbitt \\ NASA Glenn Research Center \\ Extended abstract submitted to 48th AIAA/ASME/ASCE/AHS/ASC Structures, Structural \\ Dynamics, and Materials Conference
}

The Columbia accident has focused attention on the critical need for on-orbit repair concepts for wing leading edges in the event that potentially catastrophic damage is incurred during Space Shuttle Orbiter flight. The leading edge of the space shuttle wings consists of a series of eleven panels on each side of the orbiter. These panels are fabricated from reinforced carbon-carbon (RCC) which is a light weight composite with attractive strength at very high temperatures. The damage that was responsible for the loss of the Colombia space shuttle was deemed due to formation of a large hole in one these RCC leading edge panels produced by the impact of a large piece of foam. However, even small cracks in the RCC are considered as potentially catastrophic because of the high temperature re-entry environment. After the Columbia accident, NASA has explored various means to perform on-orbit repairs in the event that damage is sustained in future shuttle flights. Although large areas of damage, such as that which doomed Columbia, are not anticipated to re-occur due to various improvements to the shuttle, especially the foam attachment, NASA has also explored various options for both small and large area repair. This paper reports one large area repair concept referred to as the "metallic over-wrap."

Environmental conditions during re-entry of the orbiter impose extreme requirements on the RCC leading edges as well as on any repair concepts. These requirements include temperatures up to $3000^{\circ} \mathrm{F}\left(1650^{\circ} \mathrm{C}\right)$ for up to 15 minutes in the presence of an extremely oxidizing plasma environment. Figure 1 shows the temperature profile across one panel (\#9) which is subject to the highest temperatures during re-entry. Although the RCC possesses adequate mechanical strength at these temperatures, it lacks oxidation resistance. Oxidation protection is afforded by converting the outer layers of the $\mathrm{RCC}$ to $\mathrm{SiC}$ by chemical vapor deposition (CVD). At high temperatures in an oxidizing environment, the $\mathrm{SiC}$ layer forms a protective $\mathrm{SiO}_{2}$ scale. However, CVD processing to form the $\mathrm{SiC}$ layer can result in the formation of small cracks in the outer surface. Hence, as a final fabrication step, a sodium silicate glass, known as "Type A," is applied as a sealant to fill any surface porosity and/or cracks in the coating and the outer portions of the RCC[1]. At relatively low temperatures, the Type A glass melts and flows into the cracks providing oxidation protection at the higher temperatures. In addition, the Type A coating, provides a "dark" coating with a high emissivity. This high emissivity allows the RCC to transfer heat by radiating outward to space as well as dispersing heat within the leading edge cavity. Lastly, the Type A possesses low catalycity which reduces surface temperatures by limiting oxygen recombination on the surface during re-entry[2]. 

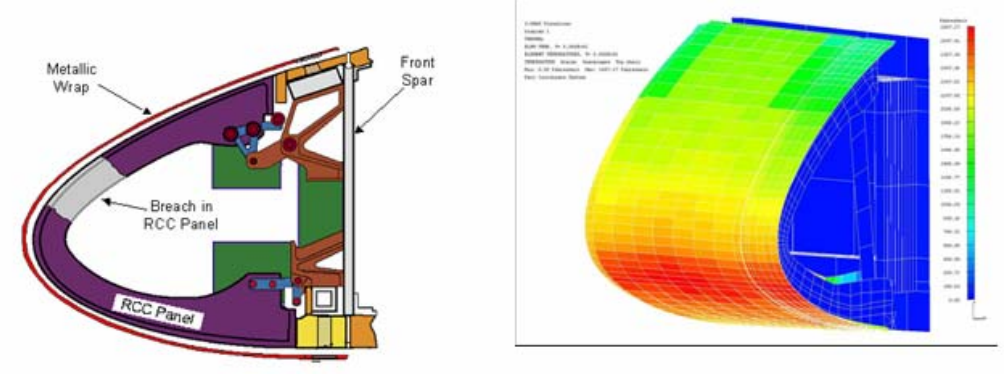

Figure 1. Over-wrap concept for leading edge wing repair and corresponding temperature profile for a single panel, $[3,4]$

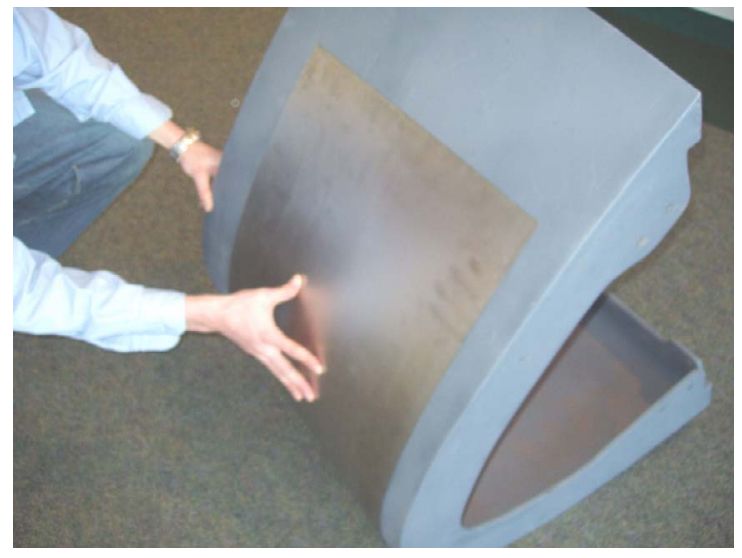

Figure 2. Metallic over-wrap concept

One proposal for on-orbit repair of the RCC leading edges is a metallic over-wrap concept (Figure 1) that would use a refractory metal sheet flexible enough to conform to the contours of the orbiter to prevent any hot gas penetration into a damaged panel cavity. This concept is illustrated in Figure 2. The metallic over-wrap concept is attractive due to its ability to cover large holes or areas of damage as well as its versatility (capability to conform to any leading edge contour thus requiring few repair sheets per flight). The objective of the current work was to develop and characterize high-temperature refractory metals that were capable of providing protection over a damaged area on the shuttle leading edge during re-entry into the earth's atmosphere.

Refractory metals, which by definition have a melting temperature greater than $3630^{\circ} \mathrm{F}$, were candidates for the flexible metallic over-wrap repair concept due to their high strength at high temperatures. The significant problem with these materials, however, is their poor oxidation resistance requiring a protective coating for high temperature use in oxidizing environments. The most effective coatings for protecting refractory metals are silicides which form a slowgrowing $\mathrm{SiO}_{2}$ scale at high temperatures in oxidizing environments [5]. Silicide coatings, however, are inherently brittle and often contain fine cracks after the coatings are applied. For the current over-wrap repair concept, bending the silicide-coated refractory sheet to conform to the contour of the leading edge could further increase cracks within the coating. 
Refractory metals and alloys based on $\mathrm{Mo}, \mathrm{Nb}, \mathrm{Ta}$, and $\mathrm{Re}$ were examined as potential over-wrap candidates because of availability and sufficient mechanical property data available for these alloys. A silicide coating, referred to as R512E (HiTemco, Old Bethpage,NY), was applied to most of the metal/alloy candidates. Although past studies exist detailing microstructure and oxidation performance of silicide coatings on many refractory alloys, no prior work on silicide coating of $\mathrm{Re}$ was found. However, stable $\mathrm{Re}$ silicides exist $\left(\mathrm{Re}_{2} \mathrm{Si}, \mathrm{ReSi}_{1.8}\right)$ which indicates the possibility to form suitable silicide layers with the R512E coating process. In addition, Re has been demonstrated as effective for reaction control system thrusters where high temperatures and oxidation resistance are required [6]. In that application, an Ir coating is used to provide environmental resistance for the Re. Hence, a thin Ir coating was also considered for oxidation protection of the Re metal. For additional protection for all of the coated alloys, the sodium silicate "Type A" coating was applied over the coatings (primarily R512E silicides) to help fill cracks and to increase the emissivity while decreasing the catalycity of the surface. In addition to the thin Ir coating, the Re sheet was also tested with the R512E silicide coating and with a thin Ir inner coating beneath an outer R512E silicide coating.

Coated alloys were screened by heating in a propane-oxygen torch that simulated the temperature profile during a 15-minute re-entry. Alloy candidates which survived this test were down-selected for arc-jet testing which more closely simulates both the temperature and the plasma environment encountered during re-entry. The Re and the Ta-10W alloys coated with $\mathrm{R} 512 \mathrm{E}$ and Type A silicate performed best (i.e., survived burn-through) during the 15 minute torch exposure. Both of these alloys also possess attractive mechanical properties. However, constraints on the number of arc-jet candidates required a further down-select. Based on its higher strength at temperatures exceeding $1500^{\circ} \mathrm{C}$, Re was chosen as the most promising refractory metal candidate for further study.

Both flat and pre-bent Re samples were arc-jet tested at Boeing LCAT. Pre-bending consisted of bending the test samples prior to coating, then flattening after coating to simulate the bending of a coated sheet to fit the contour of the leading edge. All three Re concepts were arc-jet tested and survived the 1000 second profile. Figure 3 shows the Re + R512E + Type A coating combination before and after arc-jet testing. The gray coating is the Type A sodium silicate layer. Although there is some attack obvious on the front side of the coating, it is significant that no "burn-though" occurred with these coating combinations since burn-through was typical for many competing repair concepts evaluated in the arc-jet. The cross section in Figure 4 shows that the silicide coating layer was partially consumed during arc jet testing, but the Re substrate was essentially intact. The coupons with the Ir/silicide/type A triple layer also performed well.

In conclusion, Re has proven to be a promising candidate as a metallic substrate for the repair of Space Shuttle Orbiter leading edges. An R512E silicide + Type A sodium silicate sealant coating was chosen that produces a protective, layered coating on the surface of the Re that allowed the material to survive over $1650^{\circ} \mathrm{C}$ for over 15 minutes in the oxidizing plasma environment. Arc-jet tests confirmed that this coating concept can survive the re-entry environment. The repair concepts containing an Ir layer also exhibited promising arc-jet performance but are less mature at this time, as the emissivity of Ir needs to be addressed without excessive increases to the thickness of the coated sheet. 


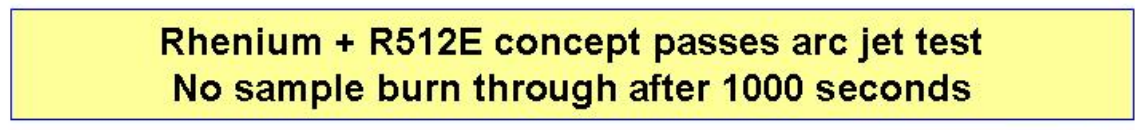

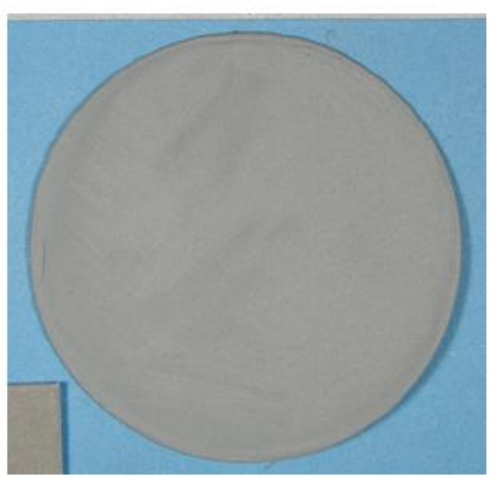

Rhenium with

Type A coating (pre-test)

Figure 3. Arc-Jet Specimens

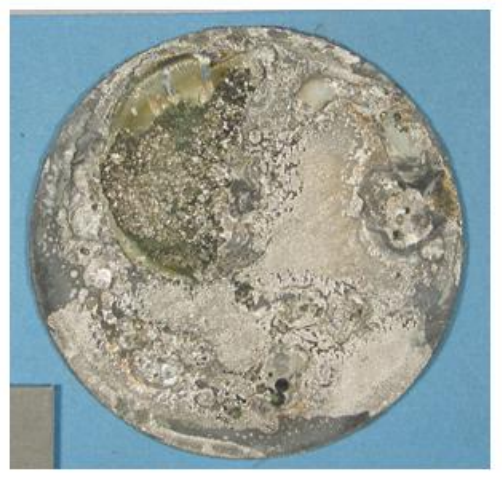

Post arc jet test (frontside)

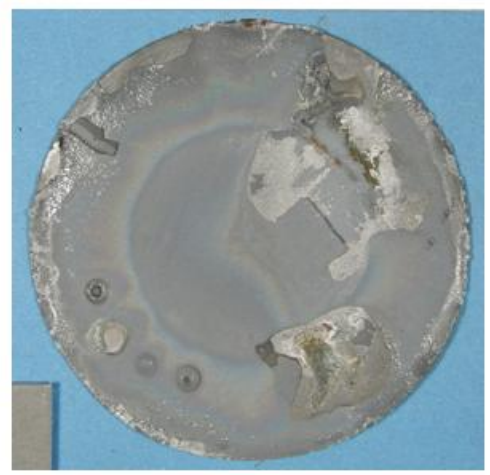

Post arc jet test

(backside)

Figure 4. Cross section

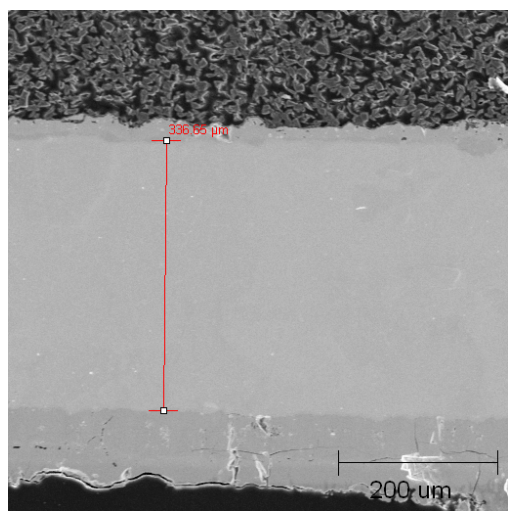
of Re/R512E, post arcjet test

\section{References}


1. M.P. Gordon; "Leading Edge Structural Subsystem and Reinforced CarbonCarbon Reference Manual", Boeing KL0-98-008, October 19, 1998.

2. D.M. Curry, J.W. Latchem, G.B. Whisenhunt, AIAA-83-0483, AIAA $21^{\text {st }}$ Aerospace Sciences Meeting, Reno, NV, January 10-13, 1983, Reno, NV.

3. M. Smith, A. Yousefiani, Z. Royzen; “On-Orbit RCC Foil Wrap Repair”, internal communication, The Boeing Co., Huntington Beach, CA, March 9, 2004.

4. F. Ghahyasi; "Return-to-Flight (RTF) RCC On-Orbit Repair Thermal Analysis Tools, Boeing internal communication, The Boeing Co., Huntington Beach, CA, January 6, 2004.

5. C.M. Packer and R.A. Perkins; "Modified Fused Silicide Coatings for Tantalum (Ta-10W) Reentry Heat Shields", NASA CR-120877, NASA Lewis Research Center, Cleveland, OH, April 1973.

6. B.D. Reed: "Long Life Testing of Oxide-Coated Iridium/Rhenium Rockets", NASA TM 107054, NASA Lewis Research Center, Cleveland, OH, July 1995. 